\title{
Application of Edge Detection Technique for Surface Roughness Estimation of Ti-6Al-4V Turned Surfaces
}

Vishwanatha J. S., Srinivasa Pai P.

Department of Mechanical Engineering, NMAM Institute of Technology, Nitte, India, E-mail: vishwajs@nitte.edu.in

In this research work, a heuristic method based on biologically motivated Particle Swarm Optimization (PSO) has been proposed for edge detection using multiresolution decomposition, to enhance the quality of the images for predicting surface roughness parameter $\mathbf{R}_{\mathrm{a}}$ from Ti-6Al-4V turned surface images. First level Dual Tree Complex Wavelet Transform (DTCWT) is used to decompose the turned images to generate new sub band images. The performance of DTCWT with PSO method is examined for turned surface images and compared with conventional edge detectors like Canny, and Sobel methods along with Discrete Wavelet Transform (DWT) with PSO and DTCWT without edge detection. The obtained results showed that, DTCWT with PSO based edge detection provides better looking edges and also best results are obtained in terms of Root Mean Square Error (RMSE) and Peak Signal to Noise Ratio (PSNR). Further, statistical features have been extracted from the images subjected to proposed edge detection method. The extracted statistical features along with machining parameters and tool flank wear have been given as inputs to radial basis function neural network (RBFNN) to predict $R_{a}$ of the turned surface images.

Keywords: Surface Roughness, Machine vision, Particle Swarm Optimization (PSO), Dual Tree Complex Wavelet Transform (DTCWT), Radial Basis Function Neural Network (RBFNN).

\section{Introduction}

The surface roughness is a main and primary indicator of the quality of machined work pieces. Generally, Titanium and its alloys are used in the manufacturing of parts and components for Biomedical, Aerospace, Automotive and other various areas of engineering $[1,2]$. Ti-6Al-4V is viewed as a challenging to machine material because of its lower heat conductivity, lower modulus of elasticity, higher chemical reactivity, and also higher corrosion resistance [3]. Surface roughness measurement using stylus approach does not support $100 \%$ assessment due to low speed and its own intrinsic constraints $[4,5]$. Optical techniques like machine vision combined with image texture analysis show better ability for non contact roughness estimation. The implementation of these methods for surface roughness assessment has drawn research attention nowadays [6, 7]. In many research works, efforts have been made for prediction of actual surface roughness through the analysis of image texture. Many image processing and machine vision applications have made significant utilization of edge detection techniques for information extraction. There are several methods proposed for quantitative image texture assessment. The edge based methods are also considered for image texture analysis, which works according to the quantity of edges in a specific region of the image $[8,29]$. Edges are connected to the pixels where there is an abrupt change in intensity [26]. Despite the ability of the edge based methods to assess image texture, they did not receive adequate attention. It has been noted that edge based features are significantly less sensitive to light intensity, which is a desirable property in industrial environments [27].

Several edge detection methods are available for locating edge pixels in an image texture. Out of which, Sobel, Canny, Laplacian of Gaussian, and Prewitt are widely used [9]. Most of these edge detectors are gradient based methods. The biologically inspired methods that are heuristic optimization techniques like, Particle Swarm Optimization (PSO), Bee Colony Optimization (BCO) and Ant Colony Optimization (ACO), have been used for edge detection [10-12]. One of the usual issues with traditional edge detectors is the use of a tiny size kernel to locate operators in a small and restricted region to identify boundary of the object inside the image. The detection quality is highly impacted by the area and size being observed, as the continuing edges of an object may take up a large part of the image. Smaller the area, the sensitivity to noise is more and lower is the localization accuracy.

In this work, a heuristic method is suggested in which the entire image is used to find for edges, without being localized. The multiresolution edge detection using PSO is carried out. First level DTCWT is used to decompose the input images as sub band images. PSO is adopted with an objective function for 
finding edges within the image. It is applied to each sub band images of DTCWT. The inverse of the processed sub band images has been taken before applying the automatic thresholding to reconstruct the final edge detectors.

Artificial intelligence is widely used in prediction of surface quality. Multilayer perceptron neural networks (MLPNN) which work on back propagation algorithm has been successfully used by many researchers in surface roughness prediction of turned surfaces [13, 25]. Apart from MLPNN, the RBFNN is one more common ANN model to improve approximation. RBFNN has great ability to compute nonlinear relations between inputs and outputs, and training is quicker than multi-layer perceptron [28].

In this work, the statistical features were extracted from the proposed edge detection method. The extracted statistical features along with machining parameters and tool flank wear considered as inputs to RBFNN to predict $R_{a}$ of the turned surfaces images. This paper is an attempt in applying multiresolution based edge detection for processing images in machining applications to improve its quality and facilitate extraction of good quality features, which provide good prediction results using any modeling technique.

\section{Experimental details}

Ti-6Al-4V round bars of dimensions $200 \mathrm{~mm}$ in length and $50 \mathrm{~mm}$ in diameter were used for machining experiments using HMT made Stallion $100 \mathrm{SU}$ $\mathrm{CNC}$ turning centre. For machining experimentation, coated carbide tool inserts of manufacturer grade 883 with MR4 chip breaker and tool holder PCLNL 2020 K12 was used (SECO TOOLS). The experiments were conducted using 150, 175 and $200 \mathrm{~m} / \mathrm{min}$ cutting speed; $0.15,0.2$ and $0.25 \mathrm{~mm} / \mathrm{rev}$ feed rate and $0.8,1$, and $1.2 \mathrm{~mm}$ depth of cut. MINITAB 17 is used for Design of experiments (DOE) methodology for planning the machining tests [13]. The best suitable DOE configuration is 33 factorial design, with a total of 27 set of experiments. The experimental setup is shown in Fig.1.

For capturing the turned images, a simple computer vision system has been designed. It includes a Sony DSC H300 digital camera (Resolution 20.1 Megapixels) for image capturing, a work table for supporting the workpiece, an adjustable tripod stand, and lighting arrangement. Turned round bars were placed on a Vblock fixture, which was kept on work table. Ti-6Al$4 \mathrm{~V}$ bar is divided into 3 sections to get a turning pass of $48 \mathrm{~mm}$ and at the end of each pass, tool flank wear is recorded. Using Mitutoyo Tool Maker's Microscope (TM 505/510) tool flank wear is measured. It has a provision for measurement along $\mathrm{X}$ and $\mathrm{Y}$ direction using micrometers. The least count of Tool Maker's Microscope is $0.005 \mathrm{~mm}$ with $15 \mathrm{X}$ magnification. The tool insert was fixed back onto the tool holder for further machining after the measurement was completed. This system was pursued until the tool flank wear attained a maximum value of $0.4 \mathrm{~mm}$. Taylor Hobson Taly Surf 50, a stylus type instrument used for measuring $R_{a}$ with $2.5 \mathrm{~mm}$ sampling length. On the surface of the workpiece at 3 different positions $120^{\circ}$ apart $R_{a}$ is measured. Finally, average value is recorded. For each pass three images are captured, total experiments consisted of 461 machining passes. Therefore a total of 1383 turned images have been considered for analysis. The camera is located at $25 \mathrm{~cm}$ distance from the surface of the workpiece and this distance is maintained constant throughout the experiments. Using Picasa tool, the captured images are cropped to pixel size 256x256. Fig. 2 shows the simple computer vision system.

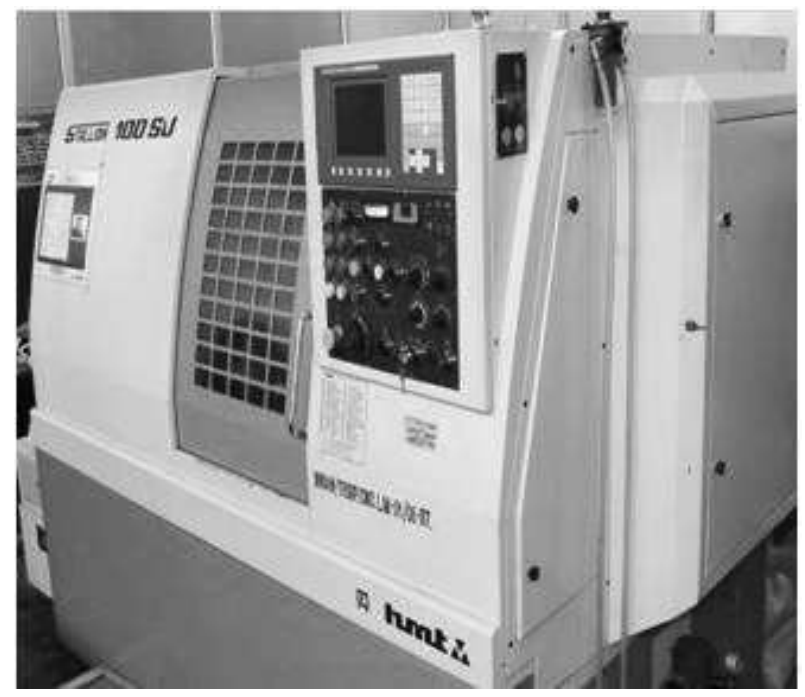

a)

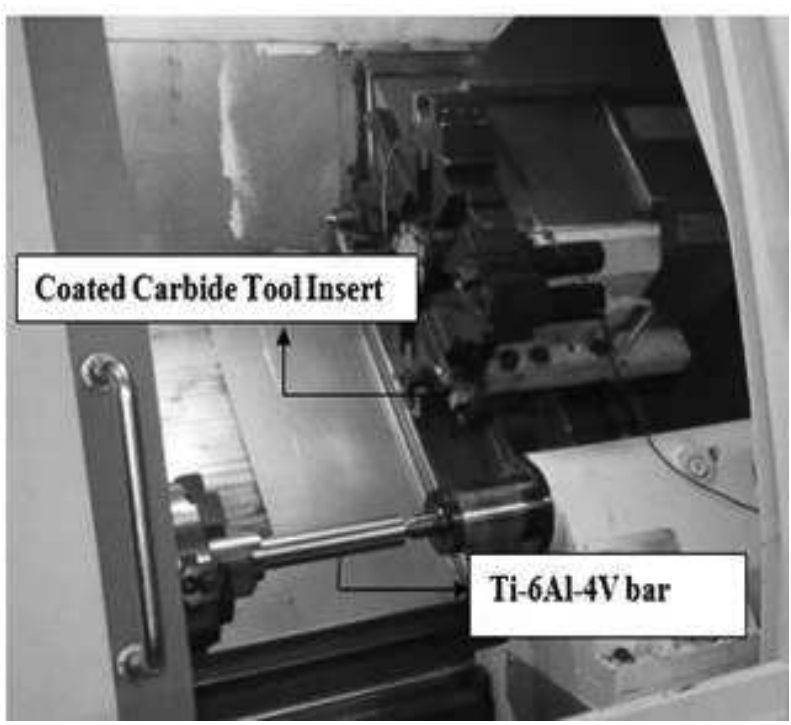

b)

Fig. 1 a) CNC Turning Centre HMT Stallion 100 SU, b) Experimental setup 


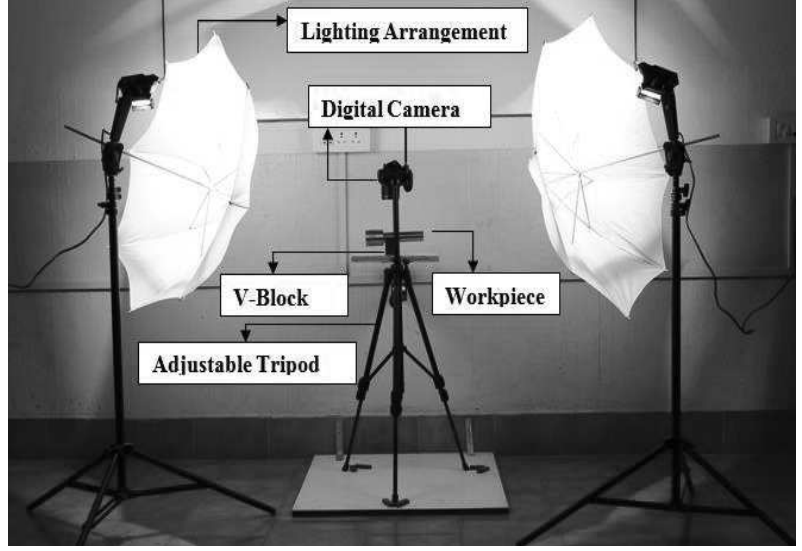

Fig. 2 A simple computer vision system

\section{Wavelet Transform}

Grossmann and Morlet [14] introduced the mathematics of wavelets to be used as a tool in signal processing. In a different way, Discrete Wavelet Transform (DW') displaces Fourier transform's infinitely oscillating sinusoidal base functions, with a set of regional oscillating base functions known as wavelets. In the classical setting, the wavelets are expanded and shifted versions of a basic, real valued band pass wavelet $\psi(\mathrm{t})$. If correctly identified and coupled with changes of the true valued low pass scaling function $\varnothing(\mathrm{t})$, it forms an orthogonal basis function for 1-dimensional real valued continuous time signal [15]. Let $x(t)$ be a finite energy analog signal, which is decomposed into wavelets and scaling function via

$$
x(t)=\sum_{n=-\infty}^{\infty} c(n) \varnothing(t-n)+\sum_{j=0}^{\infty} \sum_{n=-\infty}^{\infty} d(j, n) 2^{\frac{j}{2}} \psi\left(2^{j} t-n\right)
$$

The scaling function $\mathrm{c}(\mathrm{n})$ and wavelet coefficients $\mathrm{d}$ $(\mathrm{j}, \mathrm{n})$ are determined using

$$
\begin{aligned}
c(n) & =\int_{-\infty}^{\infty} c(n) \emptyset(t-n) d t \\
d(j, n) & =2^{j / 2} \int_{-\infty}^{\infty} x(t) \psi\left(2^{j} t-n\right) d t .
\end{aligned}
$$

In DWT, the first step is to apply the filters to the rows of image. That generates two images, first image is a set of approximate row coefficients and the other is a set of detail row coefficients. In the second step, filters are introduced to each column of newly generated images. This method of DW'T decomposition produces four sub band images. Out of these four sub band images; one is approximation image and remaining three are detail images. The detailed coefficients represent specific scale information and orientation.

\subsection{DTCWT}

DWT suffers from shift variance, and because of down sampling, aliasing occurs. Because of this, wavelet gives a different kind of output, when there is a minor change in the input signals. This issue has been resolved by Kingsbury [16] and Selesnick et al. [17] by implementing DTCW'T, which gives advantages of shift invariance, improved directional selectivity in two dimensions, perfect reconstruction, and less redundancy. Perfect reconstruction can be obtained using DTCWT, because it utilizes 2 parallel filter bank trees with real valued image coefficients generated by a single tree. Ordinary DW'T offers selectivity for diagonal characteristics in 3 fixed paths with low discrimination, whereas DTCWT produces 12 wavelet co- efficients $(6$ real coefficients and 6 imaginary coefficients) which are oriented at angles of $\pm 15^{\circ}, \pm 45^{\circ}$, $\pm 75^{\circ}$ in 2 dimensions. A 2-D image $\mathrm{f}$ is decomposed to different scales of $\mathrm{x}^{j}$ using DTCWT as given below [18]:

$$
\begin{gathered}
x=\left\{x^{1}, x^{2}, \ldots \ldots, x^{j}, y^{j}\right\} \\
x^{j}=\left\{\begin{array}{l}
x_{\operatorname{Re}, 1}^{j}(l, k), x_{R e, 2}^{j}(l, k), \ldots . x_{R e, 6}^{j}(l, k) \\
x_{I m, 1}^{j}(l, k), x_{I m, 2}^{j}(l, k), \ldots . x_{I m, 6}^{j}(l, k)
\end{array}\right.
\end{gathered}
$$

where $x^{j}$ represents $j^{\text {th }}$ level high frequency coefficient, $y^{i}$ is last level decomposition for low frequency coefficients, $x^{j}$ includes real and imaginary coefficients for 6 directional sub bands, and $(1, \mathrm{k})$ denotes coefficient location.

\section{Particle Swarm Optimization (PSO)}

Inspired by birds flocking, Kennedy and Eberhart [19] implemented PSO to simulate behaviors of swarms in order to optimize a numeric problem iteratively. The described procedure for PSO is as follows.

Each particle of a population has the following belongings: the recent position of an inquest area $\mathrm{x}_{\mathrm{i}}$, the latest velocity $\mathrm{v}_{\mathrm{i}}$, and also better position in the inquest region $\mathrm{y}_{\mathrm{i}}$. A better region $\mathrm{y}_{\mathrm{i}}$, responds to a place in the inquest area, such as objective function $f$ supplied particle $i$ with the least calculated error. The place that generated the least error throughout $\mathrm{y}_{\mathrm{i}}$ is regarded as best global position, which is denoted as y'. Eqs. (6) and (7) provide revised best positions for local and global best positions respectively. The swarm is intended to have s particles, hence $i\{1, \ldots, s\}$

$$
y_{i}(t+1)=\left\{\begin{array}{cc}
y_{i}(t), & \text { if } f\left(x_{i}(t+1) \leq f\left(y_{i}(t+1)\right)\right. \\
x_{i}(t+1), & \text { if } f\left(x_{i}(t+1)>f\left(y_{i}(t+1)\right)\right.
\end{array}\right.
$$

$$
\begin{gathered}
\mathrm{y}^{\prime}(\mathrm{t}) \epsilon\left\{\left(\mathrm{y}_{0}(\mathrm{t}), \mathrm{y}_{1}(\mathrm{t}), \ldots, \mathrm{y}_{\mathrm{s}}(\mathrm{t})\right\},\right. \\
\mathrm{f}(\mathrm{y}(\mathrm{t}))=\min \left\{\mathrm{f}\left(\mathrm{y}_{0}(\mathrm{t})\right), \mathrm{f}\left(\mathrm{y}_{1}(\mathrm{t})\right), \ldots, \mathrm{f}\left(\mathrm{y}_{\mathrm{s}}(\mathrm{t})\right)\right\}
\end{gathered}
$$

During each loop, every particle in the group is upgraded utilizing (8) and (9). Randomly produced numbers $r_{1}$ and $r_{2}$ is considered to improve performance 
of operation. For complete measurements, $j \in\{1, \ldots$ $, n\}$, let $\mathrm{x}_{\mathrm{i}, \mathrm{j}}$ give new position, $\mathrm{y}_{\mathrm{i}, \mathrm{j}}$ is local best position and $v_{i, j}$ is updated speed for $j^{\text {th }}$ dimension of $i^{\text {th }}$ particle, $\widehat{y}_{d}(t)$ provides the best particle so far found in the whole swarm at time $t$.

$$
v_{i, j}(t+1)=w_{1} \cdot v_{i, j}(t)+c_{1} r_{1, j}\left(y_{i, d}(t)-x_{i, j}(t)\right)+c_{2} \cdot r_{2, j}\left(\hat{y}_{d}(t)-x_{i, j}(t)\right)
$$

The next particle position $\mathrm{x}_{\mathrm{i}, \mathrm{j}}(\mathrm{t}+1)$ is selected using new speed $v_{i, j}(t+1)$ to the particle's recent location $\mathrm{x}_{\mathrm{i}}(\mathrm{t})$

$$
x_{i, j}(t+1)=x_{i, j}(t)+v_{i, j}(t+1)
$$

The measured speed vector value is set up in between $\left[-\mathrm{v}_{\max }, \mathrm{v}_{\max }\right]$ for reducing the probability of a particle, which tends to leave the inquest zone. Value of $\mathrm{v}_{\max }$ is generally chosen as $k \times \mathrm{x}_{\max }$, i.e. $0.1 \leq k \leq$ 1.0 , where $\mathrm{X}_{\max }$ represents the field of the inquest zone [19]. Generally, PSO execution changes width value $w$ throughout the training time. w value decreases linearly from 1 to 0 for each run. The coefficients of acceleration, $c_{1}$ and $c_{2}$ control the distance covered by a particle in each loop.

PSO parameters: The acceleration coefficients are taken as $c_{1}=2.5, c_{2}=1.5$. The population size is selected as 30 . The number of iterations considered is 50.

\section{Methodology}

Alaa Eleyan and Muhammad S Anwar [20] proposed DWT with PSO and DTCWT with PSO multiresolution edge detection for different types of images (white water rafters, girl with painted face, lighthouse in maine, and sailboat). The proposed edge detection methods were compared with Sobel, and Canny edge detection methods. The edge detection using DWT with PSO and DTCWT with PSO gave better looking edges and provided good objective evaluation results with lower RMSE and higher PSNR compared to conventional edge detectors like Canny and Sobel. Out of these two proposed multiresolution edge detection methods, DWT with PSO showed slightly better results compared with DTCWT with PSO edge detection for different images considered. This multiresolution edge detection method is adopted in this study. Machined surface images are considered for edge detection and important statistical features can be extracted from these machined images to improve the quality of modeling and prediction.

$$
\begin{aligned}
& \mathrm{f}=\left|\mathrm{I}_{(\mathrm{i}-1, \mathrm{j}-1)}-\mathrm{Z}\right|+\left|\mathrm{I}_{(\mathrm{i}-1, \mathrm{j})}-\mathrm{Z}\right|+\left|\mathrm{I}_{(\mathrm{i}-1, \mathrm{j}+1)}-\mathrm{Z}\right|+\left|\mathrm{I}_{(\mathrm{i}, \mathrm{j}-1)-\mathrm{Z}}\right|+\left|\mathrm{I}_{(\mathrm{i}, \mathrm{j}+1)}-\mathrm{Z}\right|+\left|\mathrm{I}_{(\mathrm{i}+1, \mathrm{j}-1)}-\mathrm{Z}\right|+ \\
& \left|\mathrm{I}_{(\mathrm{i}+1, \mathrm{j})}-\mathrm{Z}\right|+\left|\mathrm{I}_{(\mathrm{i}+1, \mathrm{j}+1)}-\mathrm{Z}\right|
\end{aligned}
$$

where $|$.$| gives operator of complete value and$ $Z=I(i, j)$ is the center pixel at location $(i, j)$ of an image $I$, at which recent swarm particle is located.
DTCWT utilizes two real DWT tree for image decomposition; the real part of transform is provided by first DWT, while imaginary part is provided by second DW'T tree. These two DWT trees utilize two pairs of filter bands, which need to satisfy better reconstruction conditions. First level, DTCWT is used to decompose the original machined images. The main focus is to divide original machined images into sub bands of image coefficients and apply PSO for each sub band to carry out edge detection. Finally using inverse DTCWT, the decomposed wavelet coefficients with PSO is reconstructed. The obtained reconstructed images are thresholded to get a binary image involving detected edges using the automatic thresholding function [20]. Fig. 3 represents block diagram of the methodology used in this work.

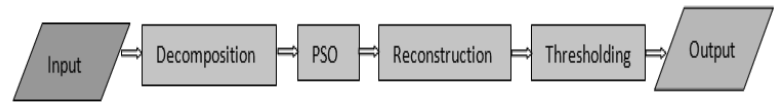

Fig. 3 Methodology of this work [20]

\subsection{PSO objective function}

Usually, an objective function is the result of the mathematical or statistical representation, which is required to be decreased or increased using nonlinear programming methods. As in all other optimization techniques, PSO also requires objective function. The performance of the algorithm is directly related to the proposed objective function. For the current work, Manhattan distance operator $\mathrm{f}$ is proposed as the objective function. The Manhattan distance operator is determined using each particle of the swarm in PSO. The particle, $p$ of a swarm of PSO finds itself on pixel c which is placed on a coordinate $(i, j)$ of image $I$. All the neighbourhood pixels around center pixels $\mathrm{c}$ are considered and the Manhattan distance from the centre pixel is determined by considering Eq. (10). Greater output of the objective function indicates higher possibility of existence of edges. Therefore, for optimization of the problem, maximization of the objective function is necessary [20].

\subsection{Automatic thresholding function}

The reconstructed images are thresholded to get binary images using automatic thresholding function. 
The automatic thresholding makes it more specific, because it acquires the output image statistical distribution, which may be different from one image to another. For DWT, the threshold $\theta_{\text {DWT }}$ is given by [20]

$$
\theta_{\text {DWT }}=\frac{\beta}{\mathrm{MN}} \sum_{\mathrm{i}=1}^{\mathrm{M}} \sum_{\mathrm{j}=1}^{\mathrm{N}} \mathrm{I}_{(\mathrm{i}, \mathrm{j})}
$$

where, $\mathrm{I}^{\prime}$ is the reconstructed image, $M \times N$ represents size of reconstructed image, and $\beta$ is the adjustable variable between 0 and 1 normally nearer to 1 .

For DTCWT with PSO method, the threshold $\theta_{\text {DTCWT }}$ is given by

$$
\theta_{\text {DTCWT }}=\beta s_{\max }
$$

where $S_{\max }$ gives pixel intensity with maximum possibility of occurrence.

\section{Results and Discussion}

\subsection{Comparison of edge detection methods}

In this work, a multiresolution edge detection technique using DTCW'T with PSO has been carried out. This method is compared with DWT with PSO, Sobel, and Canny edge detectors. Fig.4 indicates a sample original cropped image for cutting conditions: $200 \mathrm{~m} / \mathrm{min}$ speed, feed $0.2 \mathrm{~mm} / \mathrm{rev}$ and depth of cut $1.2 \mathrm{~mm}$. Fig. 5 shows the image output from different edge detection methods. From the figure, it is observed that details of rafters are much clearer for multiresolution edge detector, namely DTCWT with PSO and DWT with PSO. Sobel and Canny methods have very less edge details that make it hard to say what the image is really about. The quality measurement and assessment of edge detector is a challenging job, because edge details may be altering from one application to another. Besides human subjective examination, two objective evaluation methods i.e., Peak Signal to Noise Ratio (PSNR) and Root Mean Square Error (RMSE) are used to assess the effectiveness of the multiresolution edge detection method and comparisons have been made.

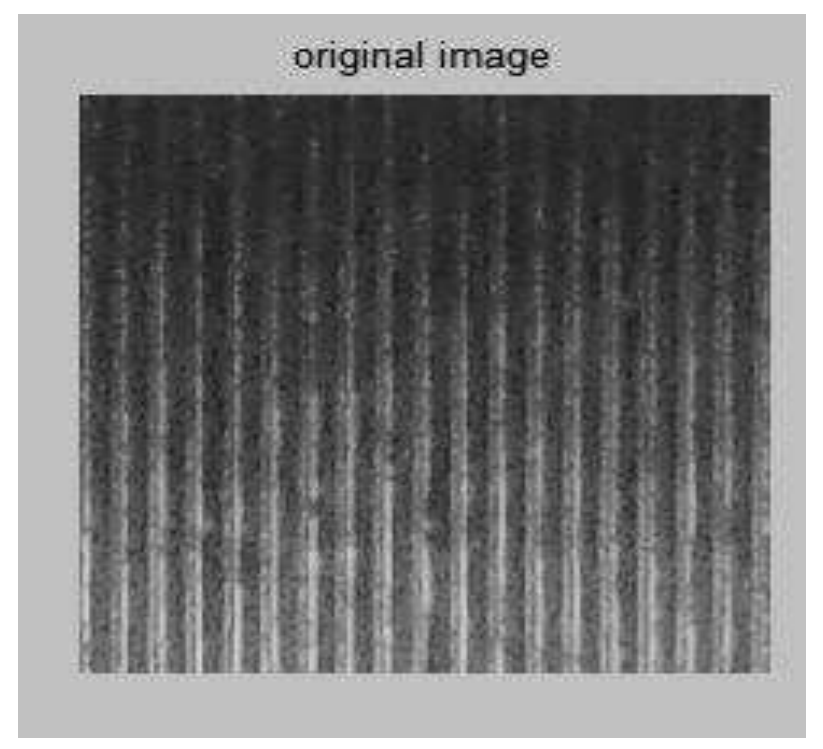

Fig. 4 Original machined image for cutting conditions: speed $200 \mathrm{~m} / \mathrm{min}$, feed $0.2 \mathrm{~mm} / \mathrm{rev}$ and depth of cut $1.2 \mathrm{~mm}$
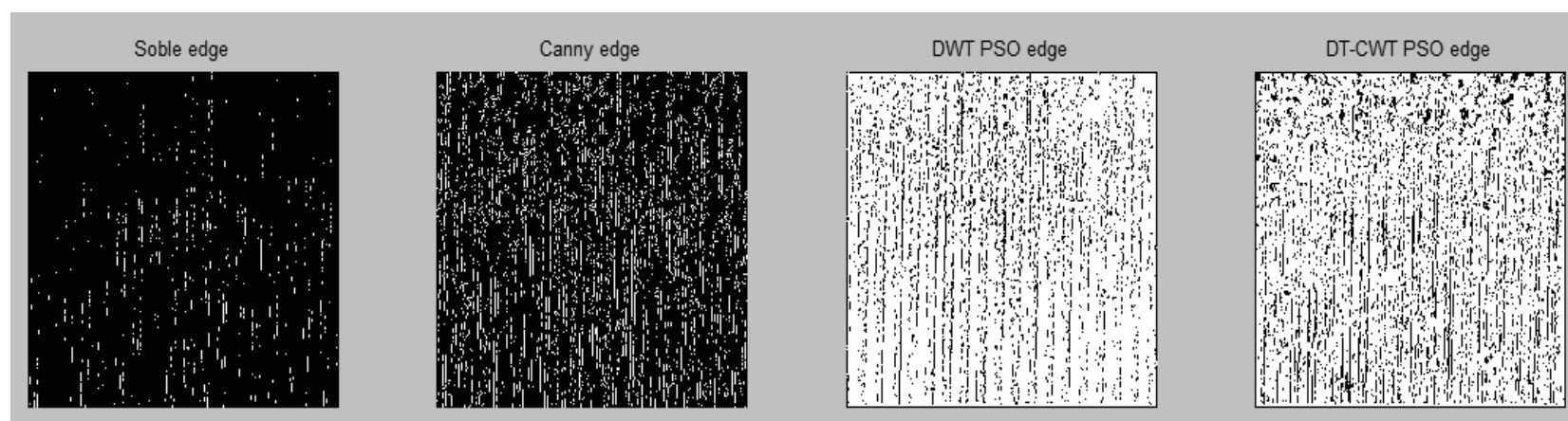

Fig. 5 Images for different edge detection methods

RMSE integrates corruption function and statistical characteristics of noise in the edge detected image. It estimates the average square difference among initial gray images and the final reconstructed binary detected edges. Higher the RMSE, larger is variance between the original and produced image. The equation for RMSE is

$$
\text { RMSE }=\frac{1}{M N} \sum_{l=1}^{M} \sum_{k=1}^{N}[R(l, k)-F(l, k)]^{2}(13)
$$

where $\mathrm{F}(1, \mathrm{k})$ is the reconstructed image after thresholding, $\mathrm{R}(1, \mathrm{k})$ is the original image, and $\mathrm{M} \times \mathrm{N}$ gives image size.
PSNR is defined as the ratio between maximum power of a signal to the power with corrupting noise, that influence the reflection quality. A PSNR with maximum value represents good quality of image [21]. It is determined using

$$
\text { PSNR }=10 \log \frac{\mathbf{2 5 5}^{\mathbf{2}}}{\mathbf{R M S E}^{\mathbf{2}}}
$$

Tab. 1 and Tab. 2 shows the PSNR and RMSE values respectively for sample images for cutting conditions: $150 \mathrm{~m} / \mathrm{min}$ speed, feed $0.15 \mathrm{~mm} / \mathrm{rev}$ and depth of cut $0.8 \mathrm{~mm}$. 
Tab. 1 PSNR values for sample machined images

\begin{tabular}{|c|c|c|c|c|}
\hline S1 no. & Sobel & Canny & DWT with PSO & DTCWT with PSO \\
\hline 1 & 42.201 & 41.499 & 47.559 & $\mathbf{4 9 . 5 4 9}$ \\
\hline 2 & 42.469 & 42.194 & 46.933 & $\mathbf{4 9 . 9 8 6}$ \\
\hline 3 & 43.198 & 42.434 & 47.796 & $\mathbf{4 9 . 0 2 7}$ \\
\hline 4 & 42.116 & 41.358 & 46.449 & $\mathbf{4 9 . 3 3 8}$ \\
\hline
\end{tabular}

Tab. 2RMSE values for sample machined images

\begin{tabular}{|c|c|c|c|c|}
\hline S1 no. & Sobel & Canny & DWT with PSO & DTCWT with PSO \\
\hline 1 & 15.738 & 14.885 & 4.717 & $\mathbf{3 . 8 4 4}$ \\
\hline 2 & 15.793 & 14.746 & 4.599 & $\mathbf{2 . 9 0 9}$ \\
\hline 3 & 15.818 & 14.848 & 5.312 & $\mathbf{2 . 6 3 0}$ \\
\hline 4 & 15.804 & 14.966 & 3.459 & $\mathbf{3 . 2 8 0}$ \\
\hline
\end{tabular}

The proposed multiresolution edge detection method is compared with traditional Canny and Sobel method in terms of PSNR and RMSE values. From Tab.1 it is observed that there has been good percentage improvement of PSNR values for DTCWT with PSO method compared with conventional Canny method for sample of machined images. Compared to Canny operator, a $16.17 \%$ increase in PSNR for DTCWT with PSO is achieved. Further, there is a reduction of $12.21 \%$ of RMSE. It is also observed that, $11.21 \%$ PSNR improvement is obtained for DWT with PSO compared with Canny edge detector and also a reduction of $11.5 \%$ of RMSE is achieved when compared with Canny operator. For the current work DTCWT with PSO edge detection method gives better results, when compared with DWT with PSO method, because DWT suffers from shift variance, aliasing and also DWT offers diagonal characteristics only in three paths, whereas DTCWT produces 12 decomposition coefficients.

In a related work, the authors have carried out surface roughness evaluation using DTCWT and GLCM extracted features from Ti-6Al-4V machined image surfaces using radial basis function neural network modeling [22, 23], where DTCWT gave better results compared to conventional DWT. The proposed multiresolution edge detection method is compared with
DWT and DTCWT without edge detection. In edge detection method, the final image is converted into binary image for proper visualization of edges. Hence it is not possible to extract the same features as described by the authors in their previous work [22, 23]. But the proposed edge detection method is compared in terms of PSNR and RMSE values. The complex decomposition of DTCW'T with PSO method retains more edges by removing unwanted, mixed and noisy edges of machined images. Tab. 3 gives the PSNR and RMSE values obtained for sample of turned images for same cutting conditions as considered in table $1 \& 2$. It is observed that, DTCWT with PSO edge detector gives better results in terms of PSNR and RMSE when compared with other edge detectors as well as DWT and DTCWT without edge detectors for evaluating turned surface images. The DWT with PSO edge detection method is compared with DW'T without edge detection and it is observed that, an average of $19.35 \%$ improvement in PSNR is achieved with a reduction of $2.85 \%$ of RMSE. Further, DTCWT with PSO edge detection method is compared with DTCWT without edge detection, it is observed that an average of $17.69 \%$ improvement in PSNR is achieved and a reduction of $2.85 \%$ of RMSE.

Tab. 3 PSNR and RMSE values for sample machined image

\begin{tabular}{|c|c|c|c|c|c|c|c|c|}
\hline \multirow{2}{*}{ S1 no. } & $\begin{array}{c}\text { DWT (without edge } \\
\text { detection) }\end{array}$ & \multicolumn{2}{c|}{$\begin{array}{c}\text { DTCWT(without } \\
\text { edge detection) }\end{array}$} & \multicolumn{2}{c|}{$\begin{array}{c}\text { DWT with PSO (with } \\
\text { edge detection) }\end{array}$} & \multicolumn{2}{c|}{$\begin{array}{c}\text { DTCWT with PSO } \\
\text { (with edge detection) }\end{array}$} \\
\cline { 2 - 9 } & PSNR & RMSE & PSNR & RMSE & PSNR & RMSE & PSNR & RMSE \\
\hline 1 & 27.807 & 7.324 & 31.426 & 6.712 & 47.559 & 4.717 & $\mathbf{4 9 . 5 4 9}$ & $\mathbf{3 . 8 4 4}$ \\
\hline 2 & 27.789 & 6.986 & 31.914 & 6.265 & 46.933 & 4.599 & $\mathbf{4 9 . 9 8 6}$ & $\mathbf{2 . 9 0 9}$ \\
\hline 3 & 27.878 & 7.729 & 32.344 & 5.892 & 47.796 & 5.312 & 49.027 & $\mathbf{2 . 6 3 0}$ \\
\hline 4 & 27.842 & 7.475 & 31.427 & 4.496 & 46.449 & 3.459 & $\mathbf{4 9 . 3 3 8}$ & $\mathbf{3 . 2 8 0}$ \\
\hline
\end{tabular}

\subsection{Surface roughness prediction using RBFNN}

Nowadays, artificial intelligence is widely used in prediction of quality of surface. The ANN model developments, genetic algorithm (GA), fuzzy logic and, information-based expert solutions implement the results in such a way similar to human beings processing information and making decisions [30]. In many fields of industrial applications artificial neural networks are used. ANN models can identify the relationship 
between input and output data sets only through learning or training through complex nonlinear inter relationships. Neural network (NN) recognizes the input and output data only by assigning the desired weights of any input variance to get the minimum error between the predicted output and the data of actual output. Multilayer perceptron (MLPs) can solve some difficult and complex problems effectively with a common supervised training algorithm known as the back propagation algorithm. Other than MLPNN, the RBFNN is one more common model of ANN for improving approximation. The RBFNN is a simple three layered feed forward neural network. The input neurons are present in first layer. The second layer consists of neurons with Gaussian function and the third layer consists of output nodes with nonlinear activation function as shown in Fig. 6. In this type of network only the weights between hidden and output layer are modified during the training. The Gaussian transfer functions have been used in the hidden layer of neurons which is given by Eq. (15).

$$
\varphi_{i}(\mu)=-\exp \left(\frac{\left\|x-\mu_{i}\right\|^{2}}{2 \sigma_{j}^{2}}\right)
$$

where $\mathrm{x}$ is the input variable, $\mu_{\mathrm{i}}$ is center variable of $\mathrm{RBF}$ unit and, $\sigma_{j}$ is variance of Gaussian function. If there are $\mathrm{N}$ number of radial basis units in the second layer and one output layer, then the output is given by Eq. (16).

$$
\mathrm{Y}=\frac{1}{1+e^{-\Sigma w_{i} \varphi+b}}
$$

where $\varphi$ signifies the activation function of the radial basis units. Weights $\mathrm{w}_{\mathrm{i}}$ defines through which the output of a RBF unit are multiplied in the output and, $b$ is a bias [24].

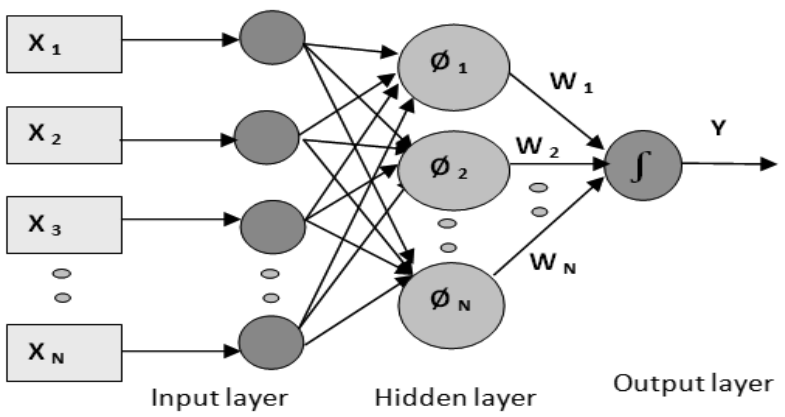

Fig. 6 A general construction of RBF neural network.

From the DTCWT with PSO edge detection method, the statistical features namely, mean, standard deviation, and variance have been extracted from the turned surface images [25]. Tab.4 shows sample of extracted statistical features from the turned surface images. The extracted features along with tool flank wear, and machining parameters namely, feed rate, speed rate, and depth of cut are considered as inputs to RBFNN model for the prediction of $\mathrm{R}_{a}$.

Tab. 4 Sample of statistical features extracted for feed $0.2 \mathrm{~mm} / \mathrm{rev}$, speed $175 \mathrm{~m} / \mathrm{min}$, and depth of cut $1.2 \mathrm{~mm}$.

\begin{tabular}{|c|c|c|c|}
\hline S1 no. & Mean & Standard deviation & Variance \\
\hline 1 & 245.77 & 47.58 & 2266.79 \\
\hline 2 & 246.08 & 46.15 & 2184.55 \\
\hline 3 & 245.87 & 47.29 & 2242.85 \\
\hline 4 & 243.20 & 53.29 & 2864.36 \\
\hline
\end{tabular}

For RBFNN modeling, out of 461 data collected from the experiments, $85 \%$ of the data (i.e. 392 data) are considered for training the model and the remaining data (69 data) considered as test data for testing the RBFNN model. The RBFNN uses the Conditional Fuzzy C- Means algorithm (CFCM) to set the number of RBF units and the position of the centers in the RBF units [13]. The CFCM algorithm is used to train the RBFNN with different widths of $0.1,0.12$, and 0.14 . The simulation parameters were kept constant namely learning rate as 0.85 , the momentum rate Tab. 5 Performance of RBFNN model for $60 \mathrm{RBF}$ units as 0.05 and maximum number of epochs as 1000 .The network has been trained by varying the RBF units; the maximum prediction accuracy is obtained for 60 RBF units. The mean squared error which is the network training goal is fixed as 0.001 . Width of the RBF units has been selected using trial and error method based on prediction accuracy. $97.42 \%$ of maximum prediction accuracy is obtained for training data and $96.52 \%$ for test data with minimum MSE for $60 \mathrm{RBF}$ units with a width of 0.12 .

\begin{tabular}{|c|c|c|c|}
\hline width & Training accuracy & Testing accuracy & MSE \\
\hline 0.1 & 96.24 & 94.44 & 0.017 \\
\hline 0.12 & 97.42 & 96.52 & 0.018 \\
\hline 0.14 & 95.95 & 94.44 & 0.018 \\
\hline 0.16 & 89.88 & 88.15 & 0.021 \\
\hline
\end{tabular}


Using Eq. (17) the percentage error for the RBFNN model was determined, which gives the relative error.

$$
\mathrm{R}_{\mathrm{e}}=\left|\left(\frac{\mathbf{V}_{\mathbf{m}}-\mathbf{V}_{\mathbf{p}}}{\mathbf{V}_{\mathbf{m}}}\right)\right| \times 100 \%
$$

where $R_{e}$ gives relative error of the model, $v_{m}$ is the actual stylus measured and $\mathrm{v}_{\mathrm{p}}$ is the predicted value of surface roughness. Tab. $\mathbf{6}$ gives the error obtained for randomly selected test data.

Tab. 6 Results obtained for test data

\begin{tabular}{|c|c|c|c|}
\hline S1. no & Experimental $\mathbf{R}_{\mathbf{a}}$ & Predicted $\mathbf{R}_{\mathbf{a}}$ & error $\mathbf{\%}$ \\
\hline 1 & 0.5194 & 0.5038 & 3.00 \\
\hline 2 & 0.3716 & 0.3823 & 2.87 \\
\hline 3 & 0.4406 & 0.4553 & 3.33 \\
\hline 4 & 0.4144 & 0.4070 & 1.78 \\
\hline
\end{tabular}

The DTCWT with PSO edge detection method provides better looking edges and it also eliminates the unwanted, mixed and noisy images. This improves the quality of the extracted features from the turned images, which further improves prediction accuracy for training and test data for the developed RBFNN model.

As discussed, the results obtained for RBFNN model have been compared with [22], in which authors have carried out surface roughness evaluation using DTCW'T and GLCM extracted features without edge detection for Ti-6Al-4V turned surface images. It has been observed that a maximum of $11.41 \%$ of increase in prediction accuracy for training data and $11.02 \%$ for test data is achieved, when compared with DTCWT with PSO edge based features. This establishes the strength of proposed edge detection method in surface roughness modeling and prediction using RBFNN.

\section{Conclusions}

The main focus of current work is to develop an edge detection method based on PSO algorithm with improved quality using DTCW'T multi resolution approach for evaluating the surface roughness of turned surface images. PSO is applied to the decomposed sub band turned images in order to get more connected edges. The DTCWT with PSO edge detection method is compared with DW'T with PSO, Sobel, and Canny edge detectors. The proposed method shows better looking edges, and further it eliminates the unwanted, mixed and noisy images. The objective evaluation in terms of PSNR and RMSE values gives better results. A comparison with DWT and DTCWT without edge detection, establishes the significance of use of edge detectors in improving the quality of turned images. DTCWT with PSO edge detection provides an average of $17.69 \%$ of PSNR improvement, with a reduction of $2.85 \%$ of RMSE values when compared with DTCWT without edge detection method.

The RBFNN model developed using DTCWT with PSO edge based features provides good results with a prediction accuracy of $97.42 \%$ for training data and $96.52 \%$ for test data. The obtained results show that the proposed RBFNN model achieves an average error rate of $2.74 \%$ for randomly selected test data, which is acceptable for this kind of applications.

\section{References}

[1] EZUGWU, E. O., BONNEY, J. AND YAMANE, Y (2003). An overview of the machinability of aero engine alloys, Journal of materials processing technology, 134(2), pp.233-253

[2] KAROL VASILKO (2019). Titanium and Technological Problems of Its Machining. Manufacturing Technology, Vol. 19, No. 3, pp.525-530

[3] GRYNALD'MELLO, P. SRINIVASAPAI, N.P. PUNEET (2017). Optimization studies in high speed turning of Ti-6Al-4V, Applied Soft Computing, 51, pp.105-115

[4] GÜRCANSAMTAŞ (2014). Measurement and evaluation of surface roughness based on optic system using image processing and artificial neural network, Int J Adv Manuf Tecbnol, 73, pp.353-364

[5] SHING I. CHANG, JAYAKUMAR S. RAVATHUR (2005). Computer Vision Based Non-contact Surface Roughness Assessment Using Wavelet Transform and Response Surface Methodology, Quality Engineering, 17

[6] V. ELANGO, L. KARUNAMOORTHY (2008). Effect of lighting conditions in the study of surface roughness by machine visionan experimental design approach. Int. J.Adv. Manuf. Technol, 37, pp.92-103

[7] P. SARMA, S. GHODRATI, S.G. KANDI, M. Mohseni (2015). A histogram-based image processing method for visual and actual roughness

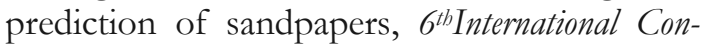
gress on Color and Coatings 2015, Tehran, Iran, pp.10-12

[8] R.N. SUTTON, E.L. HALL (1972). Texture measures for automatic classification of pulmonary disease, IEEE Trans. Comput. C-21, pp.667-676 
[9] SAJJADGHODRATI, MOHSEN MOHSENI, SAEIDEHGORJI KANDI (2019). Application of image edge detection methods for precise estimation of the standard surface roughnessparameters: Polypropylene/ethylenepropylene- diene-monomer blend as a case study, Measurement, 138, pp.80-90

[10] M. DORIGO AND S. (2006) Thomas Ant colony optimization, IEEE Computational Intelligence Magazine, 1(4), pp.28-39

[11] J. KENNEDY, RC EBERHART (2001). Swarm intelligence. Morgan Kaufmann

[12] D. KARABOGA, B. GORKEMLI, C. OZTURK, N. KARABOGA (2014). A comprehensive survey: artificial bee colony (ABC) algorithm and applications. Artificial Intelligence Review, 42(1), pp.21-57

[13] GRYNALD'MELLO, SRINIVASAPAI P, PUNEET N. P (2018). Surface Roughness Prediction in High Speed Turning of Ti-6Al-4V: A Comparison of Techniques, Materials Science and Engineering, 376

[14] A. GROSSMANN, J. MORLET (1984). Decomposition of Hardy functions into square integrable wavelets of constant shape. SIAM J. Math. Anal. 15 (4), pp.723-736

[15] IVAN SELESNICK, RICHARD BARANIUK, N.C. KINGSBURY (2005). The dual-tree complex wavelet transform, IEEE Signal Processing Magazine, 22(6), pp.123 - 151

[16] N. KINGSBURY (2001). Complex wavelets for shift invariant analysis and filtering of signals (2001) Appl. Comput. Harmon. Anal.,10 (3)

[17] I.W. SELESNICK, R.G. BARANIUK, N.C. KINGSBURY (2005). The dual-tree complex wavelet transform, IEEE Signal Process. Mag., 22 (6), pp.123-151

[18] BITING YU, BO JIA, LU DING, ZHENGXIANGCAI, QI WU, ROB LAW, JIAYANG HUANG, LEI SONG, SHAN FU (2016). Hybrid dual-tree complex wavelet transform and support vector machine for digital multi-focus image fusion. Neurocomputing, 182, pp.1-9

[19] KENNEDY, J., EBERHART, R. (1995). Particle swarm optimization. Proc. IEEE Int. Conf. Neural Netw. Perth Aust., 4, pp.1942-1948

[20] ALAA ELEYAN, MUHAMMAD S. ANWAR (2017). Multiresolution Edge Detection using Particle Swarm Optimization. International Journal of Engineering Science and Application, 1 (1)
[21] BITING YU, BO JIA, LU DING, ZHENGXIANGCAI, QI WU, ROB LAW, JIAYANG HUANG, LEI SONG, SHAN FU (2016). Hybrid dual-tree complex wavelet transform and support vector machine for digital multi-focus image fusion. Neurocomputing, 182, pp.1-9

[22] VISHWANATHA J S AND SRINIVASAPAI P (2017). DTCW' and GLCM for Surface Roughness Evaluation of Ti-6Al-4V Turned Surface using Computer Vision, Proceedings of International Conference on Manufacturing Technology and Simulation, 2017, IIT Madras, India, pp.134139

[23] VISHWANATHA J S AND SRINIVASAPAI P (2018) Modeling and prediction of surface roughness in Ti-6Al-4V turned surfaces: use of DTCWT Image Fusion and GLCM, Materials Science and Engineering, 376

[24] SIMON HAYKIN (2009). Neural networks a comprehensive foundation, second edition

[25] RAVI KEERTHI C, SRINIVASAPAI P, VISHWANATHA J S (2014). Wavelet Transform based Recognition of Machined Surfaces using Computer Vision. Applied Mechanics and Materials, pp.592-594

[26] M. SONKA, V. HLAVAC, R. BOYLE (2014). Image Processing, Analysis, and Machine Vision, 4th ed., Cengage Learning

[27] S. GHODRATI, S.G. KANDI, M. MOHSENI (2017). Roughness evaluation of randomly roughsurfaces by non-contact image profilometry method, $7^{\text {th }}$ International Congress on Color and Coatings, Tehran, Iran, December, pp.19-21

[28] SAURABH GARG, SURJYA K. PAL, DEBABRATA CHAKRABORTY (2007). Evaluation of the performance of back propagation and radial basis function neural networks in predicting the drill flank wear. Neural Comput \& Applic, Vol.16, pp.407-417

[29] A. ROSENFELD, M. THURSTON (1971). Edge and curve detection for visual scene analysis IEEE Trans. Comput. C-20, pp.562-569

[30] RIAD HARHOUT, MOHAMED GACEB, SOFIANE HADDAD, SALAH AGUIB, BENATTIA BLOUL, ADELHAMID GUEBLI (2020). Predictive Modelling and Optimisation of Surface Roughness in Turning of AISI1050 Steel Using Polynomial Regression. Manufacturing Technology, Vol. 20, No. 5, pp.591602 